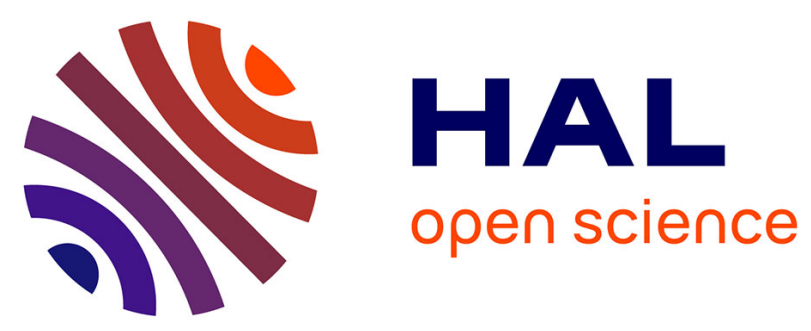

\title{
Design and experimental validation of a metacomposite made of an array of piezopatches shunted on negative capacitance circuits for vibroacoustic control
}

Flaviano Tateo, Manuel Collet, Morvan Ouisse, Mohamed Ichchou, Kenneth

A. Cunefare

\section{To cite this version:}

Flaviano Tateo, Manuel Collet, Morvan Ouisse, Mohamed Ichchou, Kenneth A. Cunefare. Design and experimental validation of a metacomposite made of an array of piezopatches shunted on negative capacitance circuits for vibroacoustic control. ASME 2013 Conference on Smart Materials, Adaptive Structures and Intelligent Systems, Sep 2013, Snowbird, United States. pp.1 - 8, 10.1115/SMASIS2013-3182 . hal-00983339

\section{HAL Id: hal-00983339 \\ https://hal.science/hal-00983339}

Submitted on 17 Jul 2018

HAL is a multi-disciplinary open access archive for the deposit and dissemination of scientific research documents, whether they are published or not. The documents may come from teaching and research institutions in France or abroad, or from public or private research centers.
L'archive ouverte pluridisciplinaire HAL, est destinée au dépôt et à la diffusion de documents scientifiques de niveau recherche, publiés ou non, émanant des établissements d'enseignement et de recherche français ou étrangers, des laboratoires publics ou privés. 


\section{DESIGN AND EXPERIMENTAL VALIDATION OF A METACOMPOSITE MADE OF AN ARRAY OF PIEZOPATCHES SHUNTED ON NEGATIVE CAPACITANCE CIRCUITS FOR VIBROACOUSTIC CONTROL}

\author{
F. Tateo, M. Collet, M. Ouisse \\ Femto-st \\ Besancon, France 25000 \\ Email: flaviano.tateo@femto-st.fr
}

\author{
M.N. Ichchou \\ Ecole Centrale de Lyon \\ Lyon, France 69134
}

\author{
K.A. Cunefare \\ Georgia Tech \\ Atlanta, USA 30332-0405
}

\begin{abstract}
In the last few decades, researchers have given a lot of attention to new engineered materials with the purpose of developing new technologies and devices such as mechanical filters, low frequency sound and vibration isolators, and acoustic waveguides. For instance, elastic phononic crystals may come to mind. They are materials with elastic or fluid inclusions inside a matrix made of an elastic solid. The anomalous behavior in phononic crystals arises from interference of waves propagating within an inhomogeneous material. The inclusions inside the matrix cause strong modifications of scattering properties. However, the application of phononic crystals is still limited to sonic frequencies. In fact, band gaps can be generated only when the acoustic wavelength is comparable to the distance between the inclusion. In order to overcome this limitation, a new class of metamaterial has been proposed: meta composite. This new class of material can modify the dynamics of the underlying structure using a bidimensional array of electromechanical transducers, which are composed by piezo patches connected to a synthetic negative capacitance. In this study, an application of the FloquetBloch theorem for vibroacoustic power flow optimization will be presented. In the context of periodically distributed, damped 2D mechanical systems, this numerical approach allows one to compute the multimodal waves dispersion curves into the entire first Brillouin zone. This approach also permits optimization of the piezoelectric shunting electrical impedance, which controls energy diffusion into the proposed semiactive distributed set of cells. Experiments performed on the examined structure illus-
\end{abstract}

trates the effectiveness of the proposed control method. The experiment requires a rectangular metallic plate equipped with seventyfive piezopatches, controlled independently by electronic circuits. More specifically, the out-of-plane displacements and the averaged kinetic energy of the controlled plate are compared in two different cases (control system on/off). The resulting data clearly show how this proposed technique is able to dampen and selectively reflect the incident waves.

\section{INTRODUCTION}

In the last few decades, researchers have given a lot of attention to new engineered materials with the purpose of developing new technologies and devices such as mechanical filters, low frequency sound and vibration isolators, and acoustic waveguides. In the field of light propagation, several efforts were made in order to design and construct photonic crystals showing photonic band gaps that prevent light from propagating in certain directions with specified frequencies. Many other efforts were made in order to create photonic crystal able to propagate light in anomalous and useful ways (i.e. negative refraction and artificial magnetism). In the acoustic domain, similar studies were carried out with the aim of preventing the propagation of elastic waves within the medium. In both cases, the band gap is obtained by periodically modulating some electromagnetic or mechanical properties [10].

This technique presents two main limitations: The spatial modulation must be of the same order as the wavelength in the gap, 
the position of the band gap cannot be easily changed since it strongly depends on the materials involved (Bragg's band gap).

A possible solution for these problems is found using composites with locally resonant units. The periodicity of the crystal creates a stop band that can be shifted by modifying the properties of the resonators. Liu et al. [7] had demonstrated that a resonant sonic crystal with building blocks of rubber-coated lead balls exhibits a low-frequency sonic band gap, and the resonance can provide a maximum impedance mismatch to shield the airborne sound. The same effect can be obtained using Helmoltz resonators as showed by Fang et al. [1,3] or $\mathrm{Hu}[6]$.

A further improvement can be obtained using shunted piezoelectric materials employed simultaneously with electronic components and controllers in order to achieve new functionalities. Adaptive metacomposite can be designed to create local resonances by coupling piezoelectric patches to a resonant external circuit, as illustrated in [9]. This concept involves a periodic array of simple RL-shunted piezos mounted on the structure to passively control the propagation of elastic waves and the subsequent vibration field. Periodically induced impedance-mismatch zones generate broader stop bands, i.e., frequency bands where waves are attenuated. The tunable characteristics of shunted piezo-patches allow the equivalent mechanical impedance of the structure to be tuned so that stop bands are generated over desired frequency ranges. The presence of a resistance in the shunt circuit generates a damped resonance of the electrical network. The resistance also allows the energy dissipation mechanism of shunted piezos to be exploited, which dampens the amplitude of vibration also outside the stop bands. In this paper, the considered metacomposite consists of several piezoelectric patches periodically arranged over the surface of the two-dimensional waveguide and shunted to an external electric circuit with a negative capacitance. Unlike the distributed resonant circuits, which are based on the energy exchange between the controlled structure and the resonant circuit, the negative capacitance circuit allows to broaden the control frequency for controlling either energy velocity or wave absorption properties. The main contribution of this article is the capacity of modifying and controlling the dynamic of the two-dimensional waveguide, in terms of reflected or absorbed energy flows. This effect is achieved by physically separating both ends of the waveguide with a periodic lattice of piezoelectric actuators.

\section{System's design}

An aluminum plate with the geometric properties listed in Table 1 is suspended to a rigid frame through metallic wires in order to reproduce the free boundaries conditions. The plate is equipped with 75 piezoelectric patches from PZ26 series (Ferroperm Industries) arranged in a regular $15 \times 5$ array (Figure 1 ). The dimension of the piezoelectric ceramics are listed in Table 1 and are justified by the following assumptions: The plate's dis-

\begin{tabular}{llll}
\hline \hline Plate & Length & 2100 & $\mathrm{~mm}$ \\
& Height & 1050 & $\mathrm{~mm}$ \\
& Thickness & 3 & $\mathrm{~mm}$ \\
& Mass density & 2700 & $\mathrm{~kg} / \mathrm{m}^{3}$ \\
& Young's modulus & $70 \cdot 10^{9}$ & $\mathrm{~N} / \mathrm{m}^{2}$ \\
& Poisson's ratio & 0.33 & - \\
\hline Piezo & Length & 50 & $\mathrm{~mm}$ \\
& Height & 50 & $\mathrm{~mm}$ \\
& Thickness & 0.5 & $\mathrm{~mm}$ \\
& Mass density & 7650 & $\mathrm{~kg} / \mathrm{m}^{3}$ \\
& Poisson's ratio & 0.31 & - \\
& Dielectic loss & $<0.05$ & $\%$ \\
Coupling factor & 0.31 & - \\
\hline \hline
\end{tabular}

TABLE 1. Geometry and physical properties of the system.

persion relation of the flexural mode at $5000 \mathrm{~Hz}$ has a wavelength of $30 \mathrm{~cm}$. This value imposes a length constraint of the piezoceramic in the propagation direction. According to Livet [8] the piezoelectric coupling is increased when the ratio between the length of the ceramics and the wavelength is bigger than $1 / 4$. Under this condition, the piezoelectric actuator can experience an acceptable strain level. The thickness of the actuator was finally defined considering the restraints of the electric circuit and the nature of the control technique. Different authors have shown that the best controlling effect is obtained when the circuit is tuned in correspondence with the biggest ceramic's capacitance value $[5,8]$. This property strongly depends on the material properties and the geometry. Once the material properties and the two dimensions of the piezoelectric actuator are chosen, the only parameter which may be amended is the thickness. Small thicknesses correspond to larger values of the intrinsic capacitance, however, this parameter cannot be reduced indefinitely due to the weakening of the piezoelectric ceramic itself. For these reasons, a thickness of $0.5 \mathrm{~mm}$ was adopted. The actual circuit's layout considered contains some passive components, such as the resistances $R_{S}, R_{2}, R_{3}, R_{4}$, the capacitance $C_{2}$ and an active component, namely an operational amplifier that actually allows the circuit to reproduce the intended behavior. This specific layout was chosen as opposed to others [4] because of its simplicity and its effectiveness in the frequency range of interest. The variation of these parameters determines the variation in the synthetic 


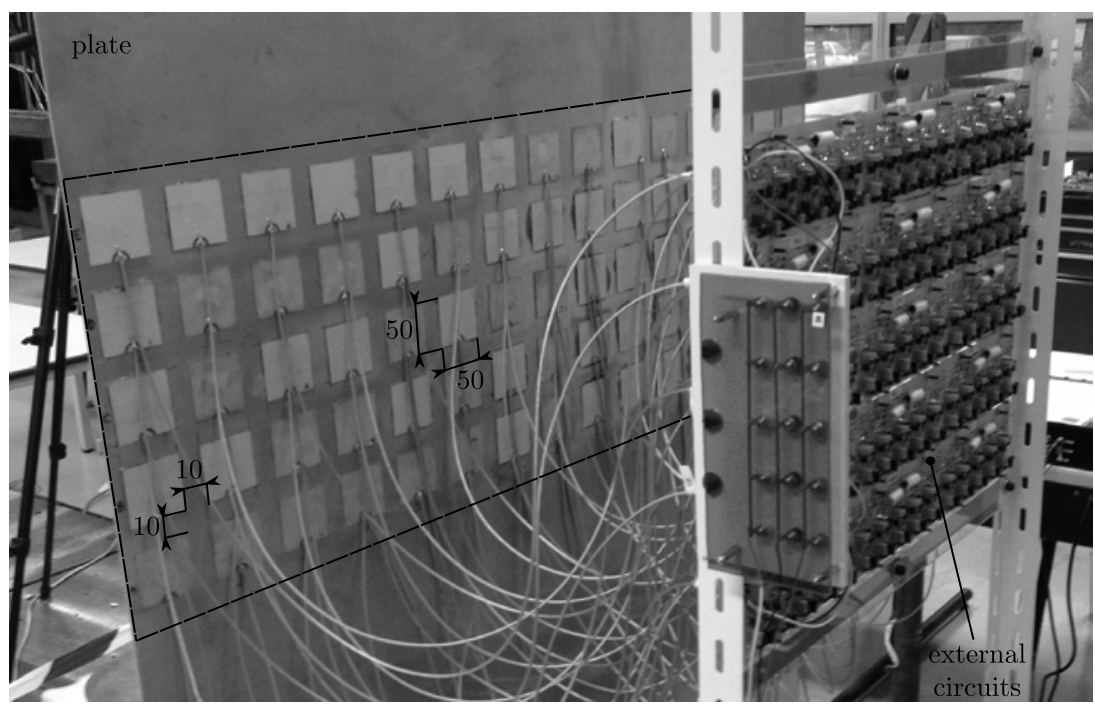

FIGURE 1. Metacomposite structure: 2D waveguide and active interface (dashed rectangle).

impedance according to the following formula:

$$
Z=R_{s}-\frac{R_{3} R_{2}}{R_{4}\left(1+i \omega R_{2} C_{2}\right)}
$$

From the practical point of view, the circuit is further modified by adding some diodes in order to protect the operational amplifier. The resistances $R_{3}$ and $R_{4}$ were merged in a single potentiometer with the aim of simplifying the circuit layout.

This control technique requires a tuning of the synthetic capacitance around the capacitance value of the piezoelectric ceramic; this value is intrinsic to the electromechanical coupling and depends upon the material properties, the geometry of the patches and the plate stiffness. The intrinsic capacitance of the piezoelectric ceramic can be easily estimated measuring the capacitance observed by an RLC-meter when the specimen is free of external forces. In the present case the measured value is 52 $\mathrm{nF}$. The actual circuit was tuned by varying the position of the potentiometer in small increments from high values of the synthetic capacitance to a value very close to the targeted intrinsic capacitance value. When the external circuit approaches the setpoint the system tends to be unstable, and an increase of voltage is observed [8]. The operational amplifier is therefore saturated. In order to mitigate this effect, the circuit's layout proposed by Forward [5] was modified adding a complementary circuit able to detect this specific instability.

\section{Experimental set-up}

The analyzed structure, shown in Figure 1, consists of an aluminum plate equipped with 75 piezoelectric actuators connected to external circuits and arranged so as to form a periodic array of $5 \times 15$ units.

The wave propagation properties of the structure are characterized by means of two-dimensional wavefield measurements, recorded with a scanning laser vibrometer (Polytec PSV-400). Measurements are conducted over a grid of $37 \times 81$ scan points with a spatial resolution of $20 \mathrm{~mm}$ in the horizontal and vertical directions. At each grid point, the laser measures the time history of the out-of-plane velocity. Elastic waves are excited by a shaker located in the lower corner of the plate, $680 \mathrm{~mm}$ away from the active interface as shown in figure 2. The transducer is driven by an input signal consisting of a random broad-band excitation and amplified by an LDS power amplifier.

\section{Scattering properties computation}

The active interface described in the previous section can be considered as a infite periodic lattice of piezo-actuators. In order to correctly describe such type of system, a general mathematical method has been developed [2]. It based on the Floquet-Bloch theorem and allows one to estimate the dispersion properties of a two-dimensional waveguide incorporating, among others, electronic components, damping effects, or any other frequencydependent characteristics.

\section{D Bloch theorem applied to piezo-elastodynamic}

Let us consider a piezoelastodynamic problem made of infinite periodic distribution of unitary cell. The harmonic homogeneous dynamical equilibrium of system is driven by the follow- 


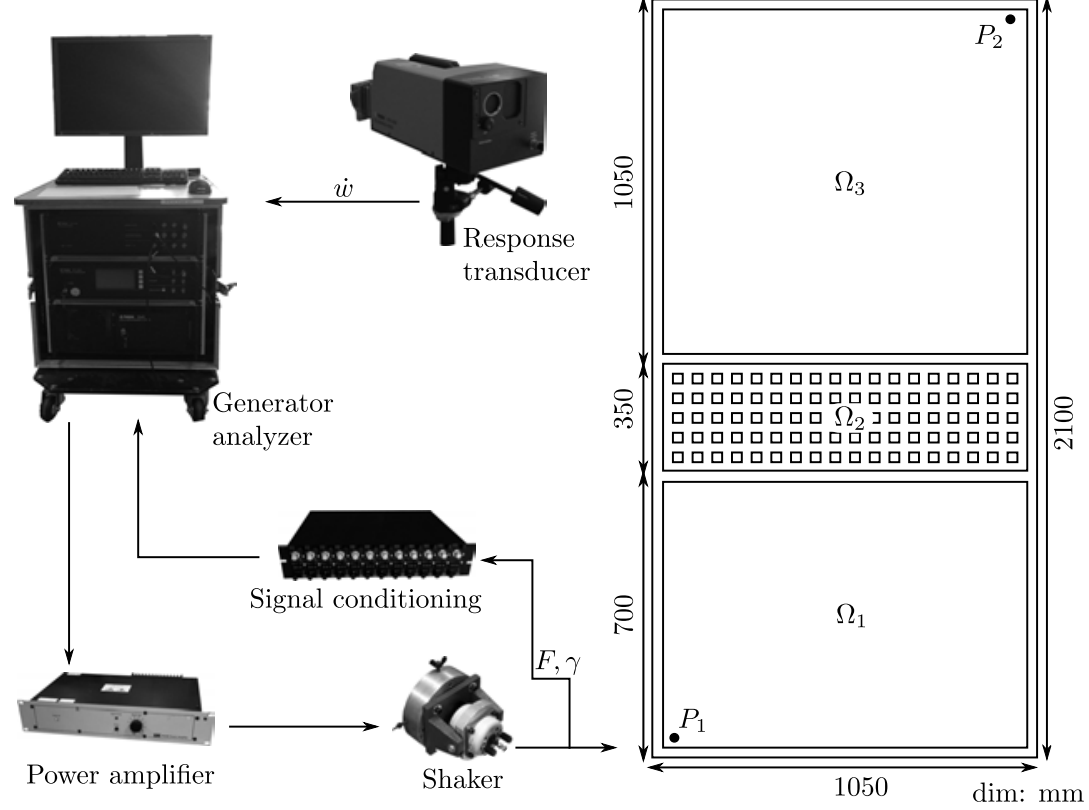

FIGURE 2. Layout of the measurement system.

ing partial derivative equations:

$$
\begin{aligned}
& \rho \ddot{w}-\nabla \cdot \sigma=0 \\
& -\nabla \cdot D=0
\end{aligned}
$$

where $w$ is the displacement vector, $\sigma$ represents the Cauchy stress tensor, $\varepsilon=\nabla_{s} w$ the Green strain tensor, and $D$ the electric displacement. The linear constitutive material behavior relationships can be written as:

$$
\begin{aligned}
& \sigma=C_{E} \varepsilon-e^{T} E \\
& D=e \varepsilon+\varepsilon_{S} E
\end{aligned}
$$

where $E=-\nabla V$ is the electric field vector ( $V$ being the voltage), $C_{E}$ the elasticity tensor at constant electrical field, $e^{T}$ the piezoelectric coupling tensor, and $\varepsilon_{S}$ the dielectric permittivity at constant strain. We add to this set of equilibrium equations an output expression

$$
q^{0}=-\int_{S_{t}} D \cdot n d S
$$

allowing the introduction of the charge measurement on the piezoelectrics top electrode and hence the dual counterpart of the imposed electrical Dirichlet boundary condition for applying the shunt impedance operator.
The equations above are consistent for each kind of material to the extent that null piezoelectric and permittivity tensors can be used when passive materials are considered. All these tensors also depend on the spatial location vector $x$ and are $\Omega_{x}$ periodic. By applying a Fourier transform, the piezoelastodynamic equilibrium can also be written as

$$
\begin{aligned}
& \rho \omega^{2} w-\nabla \cdot\left(C_{E} \nabla_{s} w+e^{T} \nabla V\right)=0 \\
& -\nabla \cdot\left(e \nabla_{s} w-\varepsilon_{S} \nabla V\right)=0 .
\end{aligned}
$$

As the problem is 2D infinitely periodic, mechanical boundary conditions are included in the formulation, while electrostatic boundary conditions have to be considered on each cell

$$
\begin{array}{ll}
V=0 & \text { on } S_{b} \\
V=V^{0} & \text { on } S_{t} \\
D_{n}=0 & \text { on } S_{l}
\end{array}
$$

where $S_{b}$ is the grounded bottom electrode of the piezoelectric layer, $S_{t}$ is the top electrode connected to the external shunt, and $S_{l}$ the lateral electrode. The top electrode applied feedback voltage $V_{0}$ depends on the shunt characteristic and on the collected charges $q_{0}$ given in equation (4) and can be expressed in the Fourier space by

$$
V^{0}=-Z q^{0}
$$


Considering a primitive cell of the periodic problem $\Omega_{x}$, the Bloch eigenmodes and the dispersion functions can be computed by searching the eigensolutions of the homogeneous problems (5) with mechanical periodic boundary conditions and electric ones given by equation (6). This solution has the form:

$$
u=u_{n, k} e^{i k \cdot x}
$$

where $u_{n, k}$ is composed of $\Omega_{x}$ periodic functions. By introducing expression (8) in the piezoelastodynamic equations, is then possible to determine the functions $w_{n, k}, V_{n, k}$, and $\omega_{n}$, solutions of the generalized eigenvalues problem

$$
\begin{aligned}
0= & \rho \omega^{2} w_{n}-\nabla \cdot\left(C_{E} \nabla_{s} w_{n}+e^{T} \nabla V_{n}\right)+ \\
& i k\left(\left(C_{E} \nabla_{s} w_{n}\right) \cdot \Phi+\nabla \cdot\left(C_{E} \Xi_{n, k}\right)\right)+ \\
& i k\left(\left(e^{T} \nabla V_{n}\right) \cdot \Phi+\nabla \cdot\left(e^{T} \nabla V_{n}\right) \cdot \Phi\right)+ \\
& -k^{2}\left(\left(C_{E} \Xi_{n, k}\right) \cdot \Phi+\left(e^{T} \nabla V_{n} \cdot \Phi\right) \cdot \Phi\right)+ \\
0= & -\nabla \cdot\left(e \nabla_{s} w-\varepsilon_{S} \nabla V\right)+ \\
& -k\left(\nabla \cdot\left(e \Xi_{n, k}\right)+\left(e \nabla_{s} w\right) \cdot \Phi\right)+ \\
& +k\left(\nabla \cdot\left(\varepsilon_{S} \nabla V\right) \cdot \Phi+\left(\varepsilon_{S} \nabla V\right) \cdot \Phi\right)+ \\
& +k^{2}\left(\left(e \Xi_{n, k}\right) \cdot \Phi-\left(\varepsilon_{S} \nabla V \cdot \Phi\right) \cdot \Phi\right)+ \\
&
\end{aligned}
$$

with the associated boundary conditions

$$
\begin{array}{ll}
w_{n, k}^{\text {out }}=w_{n, k}^{\text {in }} & \text { on } S_{R}, S_{T} \\
V_{n, k}=0 & \text { on } S_{b} \\
V_{n, k}=-Z q_{n, k} & \text { on } S_{t} \\
D \cdot n=0 & \text { on } S_{l}
\end{array}
$$

In these equations $\underline{k}=\Phi k$ where $\Phi=[\cos \theta, \sin \theta, 0]^{T}$ represents the direction angles into the reciprocal 2D lattice domain and $\Xi_{n, k}=1 / 2\left(w_{n, k} \cdot \Phi^{T}+\Phi \cdot w_{n, k}^{T}\right)$ is the symmetric dyadic tensor or the dyadic product of the displacement $w$ and direction vector $\Phi$. $S_{R}$ are the interfaces of the cells. In the electrical boundary conditions, $q^{0}$ is given by

$$
q_{n, k}^{0}=\int_{S_{t}}\left(-e \nabla_{s} w+i k \nabla e \Xi+\varepsilon_{S}(\nabla V+i k V \cdot \Phi)\right) \cdot n d S
$$

\section{Numerical computation of the Blochs waves}

The numerical implementation is obtained by using a standard FE method to discretize the piezoelastodynamic equations formula. The assembled matrix equation is given by

$$
\left(K(Z)+\lambda L(\theta, Z)-\lambda^{2} H(\theta, Z)-\omega^{2} M\right) u(\theta, k)=0
$$

where $\lambda=i k ; M$ and $K$ are the standard symmetric semidefinite mass and stiffness matrices (the mass matrix is semidefinite because elastostatic equation is condensed into the equation), $L$ is a skew-symmetric matrix; and $H$ is a symmetric semidefinite positive matrix. $u$ is the generalized eigenvector defined on all degrees of freedom of the used FE model.

When $k$ and $f$ are fixed and $Z$ does not depend on $\omega$, the system (12) is a linear eigenvalue problem allowing us to compute the dispersion functions $\omega^{2}$ and the associated Bloch eigenvector $u_{n}$. This approach has been widely used for developing homogenization techniques and spectral asymptotic analysis.

Nevertheless, these approaches have been only developed for undamped or lightly damped mechanical systems. In these cases, most of the previously published works present techniques based on the mesh of a real $k$-space following the boundary of the first Brillouin zone for obtaining the corresponding dispersion curves and the associated Floquet vectors. For undamped system, only propagative or evanescent waves exist corresponding to a family of eigensolutions purely real or imaginary. Discrimination between each class of waves is easy. If a highly damped system ( $K$, $L$, and $H$ are complex frequency dependent) and a frequencydependent electrical shunt impedance are considered, the obtained eigenvalue problem is not quadratic and specific numerical methodology has to be implemented. Furthermore, evanescent parts of propagating waves appear as the imaginary part of $\omega^{2}$. It then becomes much more difficult to distinguish the propagative and evanescent waves as all solution appear complex.

Another much more suitable possibility for computing damped system is to consider the following generalized eigenvalue problem

$$
\left(K(Z)+\lambda L(\theta, Z)-\lambda^{2} H(\theta, Z)-\omega^{2} M\right) u(\theta, \omega)=0
$$

In this problem, the angular frequency $\omega$ is a real parameter corresponding to the harmonic frequency. Wave numbers and Floquet vectors are then computed. As $L$ is skew-symmetric, the obtained eigenvalues are quadruple collapsing into real or imaginary pairs when all matrices are real (i.e. for an undamped system). In this case, a real pair of eigenvalues corresponds to evanescent modes oriented in two opposite directions on the $k$ space and imaginary values to two traveling waves propagating in opposite direction. As previously mentioned, the real part of $\underline{k}=\Phi k$ vector is restricted to stand inside the first Brillouin zone. In the quadratic eigenvalue problem (13), nothing restricts computation to only find eigenvalues satisfying this condition. For direction vector $\Phi$ orthogonal to the lattice facelets, the periodical conditions expressed for 1D wave guide are still valid. Thus, for undamped systems, all obtained eigenvalues are periodically distributed in the $k$-space along its principal directions.

\section{Group velocity and electric power estimation}

In order to correctly decribe and qualify the properties of this distributed control system some new quantities must be in- 
troduced.

The first one, which is considered for describing the capability of the metacomposite for transmitting structural energy, is the wave group velocities. They indicate how energy is transported into the considered system and allow to distinguish the propagative and evanescent waves. If a Bloch eigensolution is considered, the associated group velocity vector is given by:

$$
C_{n}^{g}=\nabla_{k} \omega=\frac{\langle\langle S\rangle\rangle}{\left\langle\left\langle e_{t o t}\right\rangle\right\rangle}=\frac{\langle I\rangle}{\left\langle E_{\text {tot }}\right\rangle}
$$

where \langle\rangle is the spatial and time average, respectively, on one cell and one period of time; $S$ is the density of energy flow; $I$ is the mean intensity; and $e_{t o t}$ and $E_{t o t}$ are the total piezomechanical energy and its time average on a period. In this problem, we only consider mechanical energy transportation as the electrostatic coupling is decentralized on each cell and cannot induce spatial energy transportation.

The intensity vector $I$ is expressed as

$$
\langle I\rangle=-\frac{\omega}{2} \operatorname{real}\left(\int C_{E}(\varepsilon+i k \Xi) w^{*} d \Omega\right) .
$$

As the spatiotemporal average of the system Lagrangian is null, the total energy average is approximated by only computing the kinetic energy average

$$
\left\langle E_{t o t}\right\rangle=\int_{\Omega} \rho w w^{*} d V
$$

The group velocity vectors $C_{n}^{g}$ are computed for all wave numbers at each frequency. In order to focus our analysis on only flexural modes ( $S$ and $S H$ ones), we introduce an indicator allowing to select them by computing the ratio of kinetic energy average on out-of-plane displacement as

$$
\operatorname{Ind}_{z}=\frac{T_{z}}{T}
$$

$T_{z}$ being the time-averaged kinetic energy in the out-of-plane direction, and $T$ the total kinetic energy. The characterization of the absorbing properties of the control system rely on the quantity of energy dissipated within the external circuit at each cycle. The damped electric power is expressed as

$$
P_{e l}=\frac{1}{2} \operatorname{real}\left(V^{0}\left(i \omega q^{0}\right)^{*}\right)
$$

\section{RESULTS AND DISCUSSION}

In this section some theoretical and experimental results are given in order to show the effectiveness of the proposed distributed control technique. First, from the theoretical point of view a comparison between the dispersion curves of the waveguide will show how the dynamic of the waveguide is influenced by the external electric circuits. In order to prove the consistency of the results for the real bounded system a full-field measurement will further illustrate the main features of the control system, characterizing the reflective and absorbing properties of the considered metacomposite.

\section{Analysis of the dispersion properties}

The propagative properties of this smart system will be analyzed comparing the dispersion diagrams, the electric power and the group velocity of the metacomposite. Three different configuration will be analyzed: open circuit, negative capacitance with reflecting (case R) and absorbing (case A) capabilities. The reflecting properties are increased setting the external circuit as follows: the capacitance have a value of $-62 \mathrm{nF}$ and a small resistance of $40 \Omega$. The damping effect is obtained by setting the external capacitance at $-62 \mathrm{nF}$ with a bigger resistance of 400 $\Omega$.

Figure 3 shows the dispersion curves using the propagative parts of the wave numbers $k_{x}$ along $(O x)$ axis. This figure shows the ability of the procedure to catch all Blochs solutions including evanescent ones that generally do not appear in literature because of their imaginary character. In the context of structural dynamics, including damping effects is mandatory for real-life applications and all wave numbers become complex. By using a suitable wave filter based on the group velocity, it is possible to retain the only propagative solutions responsible for energy transportation. It then becomes easy to observe branches similar to standard $S_{0}, A_{0}, A_{1}$, and $S H$ waves. The corresponding group velocities along $(O x)$ are presented in the same figure. It can be immediately observed that the use of the shunt impedance leads to a larger decrease of the group velocity of the $A_{0}$ modes. The bending waves also propagate energy with a very slow velocity and can be considered as evanescent. Flexural energy is, also, only transported by the $A_{1}$ mode after the cutting frequency. The propagative part of the controlled wave is strongly modified, as illustrated in Figure 3 (red line versus black line): from two complex conjugated solutions, the $A_{0}$ wave changes after control to four solutions (two complex conjugates and their opposites). It can also be observed that a nondispersive symmetric wave is also affected by the control, even if it was not targeted.

Another strategy for optimizing the adaptive metacomposite consists in focusing on the damped power flow inside the electric shunts. In this case, the objective is to improve the absorption capability of the smart structure. The propagative parts of the wave number $k_{x}$ along $(O x)$ axis appear as shown in Figure 3 
along with the corresponding group velocities along $(O x)$ and the electrically damped power. The first observation is that the optimization of the shunt impedance for improving the absorption characteristics of the system induces modifications of the group velocities of the controlled waves, while the propagative part of the wave numbers remain unchanged. This can be explained by a large improvement of the ratio between the real and imaginary parts of the wave numbers, which physically corresponds to the forcing of propagating effects to increase damping effects: energy can propagate inside the periodically distributed set of active cells for allowing electrical energy conversion, the dissipated power is largely increased when the shunt is connected to the patch (blue line).

\section{Kinetic energy distribution}

The modification of the internal dynamic of the waveguide can be proved through the estimation of the kinetic energy distribution calculated integrating the measured velocity field over the total plate's area. In particular, observing Figure 2 we can identify three different region that ideally separate the two ends of the plate and the active interface.

Figure 4 presents the kinetic energy field over the whole plate's surface. The top shows a comparison of the kinetic energy distribution among three different circuits' configuration at the tuning frequency of $2500 \mathrm{~Hz}$. On the left, (control OFF) the kinetic energy is evenly spread over the entire plate. In the middle, the system's response is slightly modified using a small resistance $R_{S}$ (case R). On the contrary, as a result of increasing the value of the resistance $R_{s}$ (case $\mathrm{A}$ ), the kinetic energy distribution of the plate is clearly modified; the waves are confined in the portion of the plate below the active interface. Considering higher frequency values, for instance $3000 \mathrm{~Hz}$, the behavior of the system is radically modified. In case $\mathrm{R}$, the vibratory energy of the plate is confined in the region $\Omega_{1}$. In case $\mathrm{A}$, the reflective behavior is no longer present, and the driving control mechanism is a pure dissipative effect that makes the kinetic energy field weaker.

\section{CONCLUSIONS}

This paper describes the analysis of wave propagation in a periodic two-dimensional waveguide with shunted piezoelectric patches. The coupling between the plate and this smart interface allows the modification of its internal dynamic. The wave propagation characteristics of the piezoelectric waveguide are first predicted through the application of the Floquet-Bloch theorem. The dispersion analysis highlights the generation of a control band where the attenuation or the reflection of the waveguide is increased. In particular selecting appropriately the external circuit's parameter is possible to reduce the group velocity of the incoming waves, that is, the active interface prevent the wave to propagate. By increasing the resistance $R_{s}$ the waveguide expe-
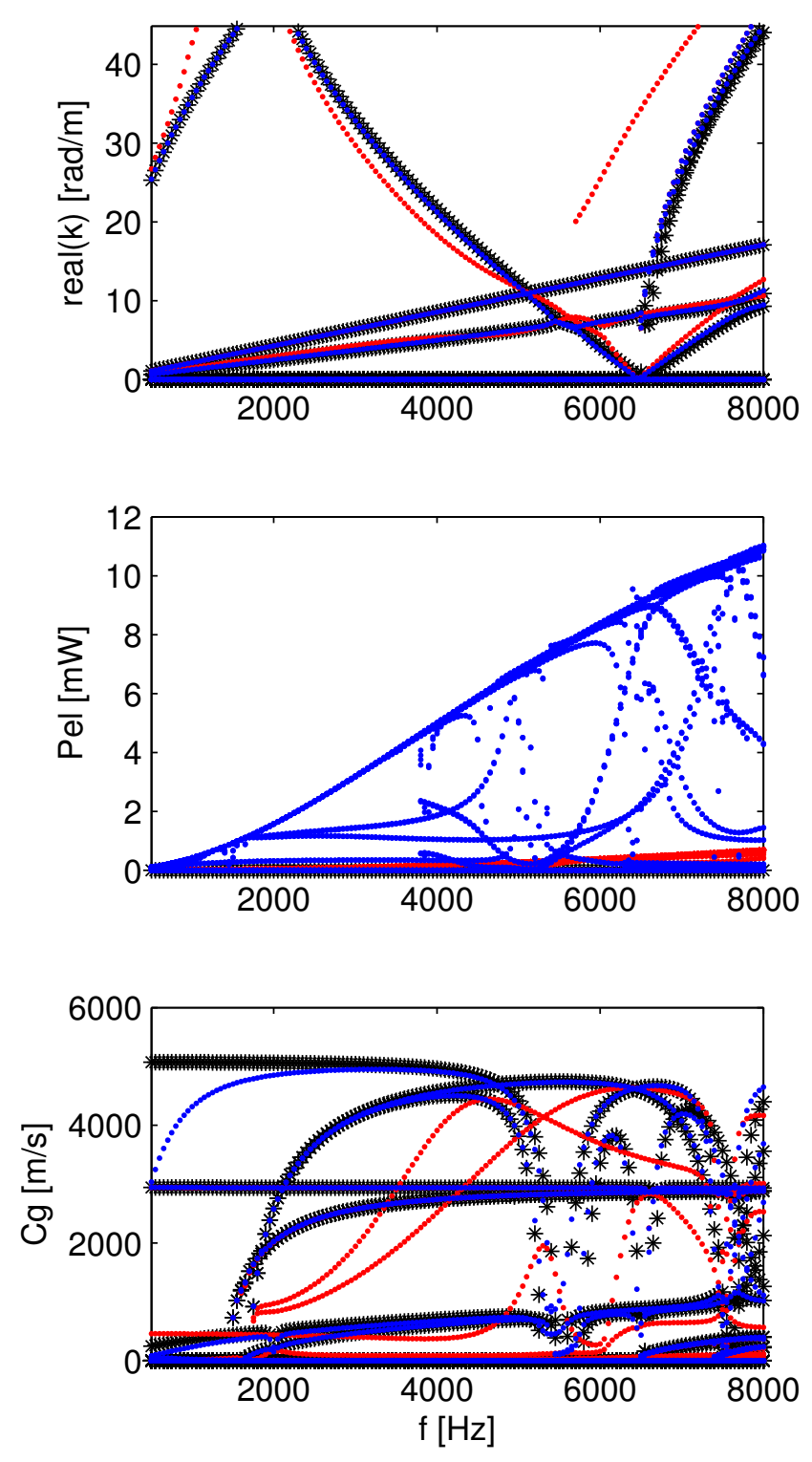

FIGURE 3. Comparison of the dispersion relation (top), dissipated electric power (middle) and group velocity (bottom) of the smart waveguide. Three cases have been considered: $O C$ (black markers), $R C_{n e g}$ case $\mathrm{R}$ (red markers) and $R C_{\text {neg }}$ case $\mathrm{A}$ (blue markers).

riences an increase of the damping over a broad frequency band. Experimental evidence of the internal controlling behavior of the waveguide is then provided through measurements of the out-ofplane velocity of the plate. The experimental results effectively confirm the numerical predictions. 


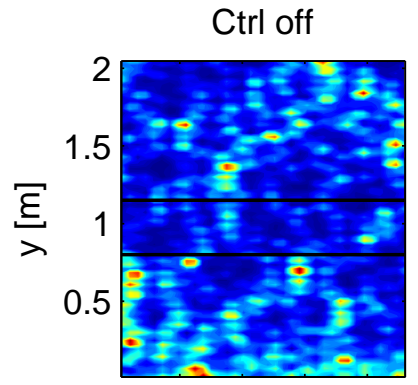

0.20 .40 .60 .8

$\mathrm{x}[\mathrm{m}]$

Ctrl off

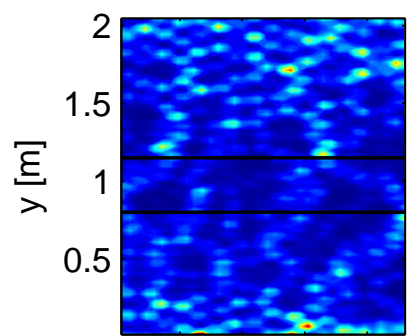

0.20 .40 .60 .8

$\mathrm{x}[\mathrm{m}]$

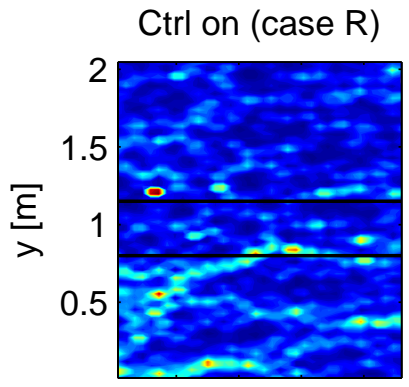

0.20 .40 .60 .8

$\mathrm{x}[\mathrm{m}]$

Ctrl on (case $\mathrm{R}$ )

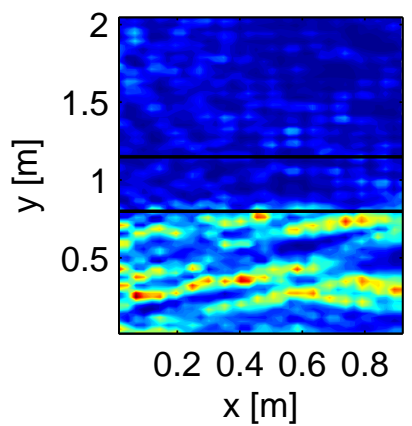

Ctrl on (case A)

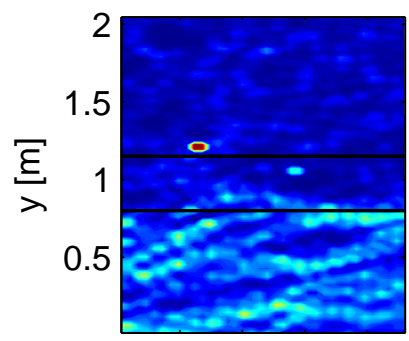

0.20 .40 .60 .8

$x[\mathrm{~m}]$

Ctrl on (case A)

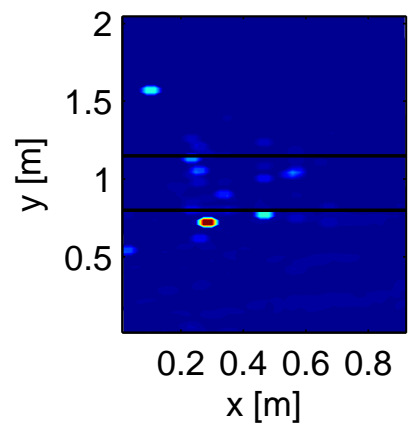

FIGURE 4. Plate's kinetic energy field: $O C$ (left), $R C_{n e g}$ case $\mathrm{R}$ (middle) and $R C_{n e g}$ case A (right). Two different frequency values have been considered $2500 \mathrm{~Hz}$ (top) and $3000 \mathrm{~Hz}$ (bottom).

\section{ACKNOWLEDGMENT}

This study is a collaborative effort supported by the French Research Agency under grant number NT09-617542 and is supported by the Labex Action.

\section{REFERENCES}

[1] M. Ambati, N. Fang, C. Sun, and X. Zhang. Surface resonant states and superlensing in acoustic metamaterials. Physical Review B, 75(19):195447, 2007.

[2] M. Collet, M. Ouisse, and M.N. Ichchou. Structural energy flow optimization through adaptive shunted piezoelectric metacomposites. Journal of Intelligent Material Systems and Structures, 23(15):1661-1677, 2012.

[3] N. Fang, D. Xi, J. Xu, M. Ambati, W. Srituravanich, C. Sun, and $\mathrm{X}$. Zhang. Ultrasonic metamaterials with negative modulus. Nature Materials, 5(6):452-456, 2006.

[4] A.J. Fleming, S. Behrens, and S.O.R. Moheimani. Synthetic impedance for implementation of piezoelectric shuntdamping circuits. Electronics Letters, 36(18):1525-1526, 2000.

[5] R.L. Forward. Electronic damping of vibrations in optical structures. Applied Optics, 18(5):690-697, 1979.
[6] X. Hu, C.T. Chan, and J. Zi. Two-dimensional sonic crystals with helmholtz resonators. Physical Review E, 71(5):556, 2005.

[7] Z. Liu, X. Zhang, Y. Mao, Y.Y. Zhu, Z. Yang, C.T. Chan, and P. Sheng. Locally resonant sonic materials. Science, 289(5485):1734-1736, 2000.

[8] S. Livet, M. Collet, M. Berthillier, P. Jean, and J.M. Cote. Structural multi-modal damping by optimizing shunted piezoelectric transducers. European Journal of Computational Mechanics, 20(1-4):73-102, 2011.

[9] O. Thorp, M. Ruzzene, and A. Baz. Attenuation and localization of wave propagation in rods with periodic shunted piezoelectric patches. Smart Materials and Structures, 10(5):979, 2001.

[10] S. Yang, J.H. Page, Z. Liu, M.L. Cowan, C.T. Chan, and P. Sheng. Ultrasound tunneling through 3D phononic crystals. Physical Review Letters, 88(10):104301, 2002. 